\title{
Application of Volume diffraction grating for terahertz lasing in Volume FEL (VFEL)
}

\author{
V.G.Baryshevsky, K.G.Batrakov, if and V.I.Stolyarsky状 \\ Institute of Nuclear Problems, Belarusian State University, \\ 11 Bobruiskaya Str., Minsk 220050, Belarus.
}

(Dated: November 4, 2018)

\begin{abstract}
The generation of induced radiation in volume resonator formed by metal threads is considered. It is shown that using of such volume diffraction grating allows increasing of lasing efficiency in terahertz range. The requirements on beam and grating parameters are obtained.
\end{abstract}

\section{INTRODUCTION}

Generation of radiation in millimeter and far-infrared range with nonrelativistic and low-relativistic electron beams gives rise difficulties. Gyrotrons and cyclotron resonance facilities are used as sources in millimeter and sub-millimeter range, but for their operation magnetic field about several tens of kiloGauss $\left(\omega \sim \frac{e H}{m c} \gamma\right)$ is necessary. Slow-wave devices (TWT, BWT, orotrons)in this range require application of dense and thin (¡0.1 mm) electron beams, because only electrons passing near the slowing structure at the distance $\leq \lambda \beta \gamma /(4 \pi)$ can interact with electromagnetic wave effectively. It is difficult to guide thin beams near slowing structure with desired accuracy. And electrical endurance of resonator limits radiation power and density of acceptable electron beam. Conventional waveguide systems are essentially restricted by the requirement for transverse dimensions of resonator, which should not significantly exceed radiation wavelength. Otherwise, generation efficiency reduces abruptly due to excitation of plenty of modes.

The most of the above problems can be overpassed in VFEL. In VFEL the greater part of electron beam interacts with electromagnetic wave due to volume distributed in-

\footnotetext{
*E-mail me at:bar@inp.minsk.by

${ }^{\dagger}$ E-mail me at:batrakov@inp.minsk.by

${ }^{\ddagger}$ E-mail me at:vist@inp.minsk.by
} 
teraction. Transverse dimensions of VFEL resonator could significantly exceed radiation wavelength $D>>\lambda$. In addition, electron beam and radiation power are distributed over the whole volume that is beneficial for electrical endurance of the system. Multi-wave Bragg dynamical diffraction provides mode discrimination in VFEL.

Dispersion equations, describing instability and threshold conditions of generation for VFEL were investigated in details in [1]-[4]. There were shown that threshold values of current can be significantly reduced in conditions of multi-wave Bragg diffraction. But there mainly relativistic and ultrarelativistic electron beams were considered. Essential distinctions appear at use of nonrelativistic and low-relativistic electrons. For example, in slow-wave devices radiation conditions can not be fulfilled simultaneously with conditions of Bragg diffraction if refraction index $n<c / u$. Generation in spatially-periodic structure composed from dielectric threads was studied in [5] for low-relativistic (300 keV)electron beam.

Present paper investigates instability of electron beam in volume diffraction structures composed from strained threads. There are frequency ranges, in which such systems can generate, and those in which they work as amplifiers. It should be mentioned that generation can occur even in one-periodic diffraction structure. In this case Bragg conditions are not fulfilled and back-wave is used for generation. Tuning of radiation frequency in such a system can be provided either by change of radiation angle or by grating rotation or by change of direction and value of electron beam velocity. Nonrelativistic and low-relativistic electron beams passing through such structures can generate in wide frequency range up to terahertz.

\section{AMPLIFICATION AND GENERATION IN DIFFRACTION STRUCTURE}

Let us consider an electron beam with velocity $\vec{u}$ passing through a periodic structure composed from either dielectric or metal threads (see Figure [.) Fields, which appear at electron beam passing through a volume spatially-periodic medium are described by the following set of equations [3]: 


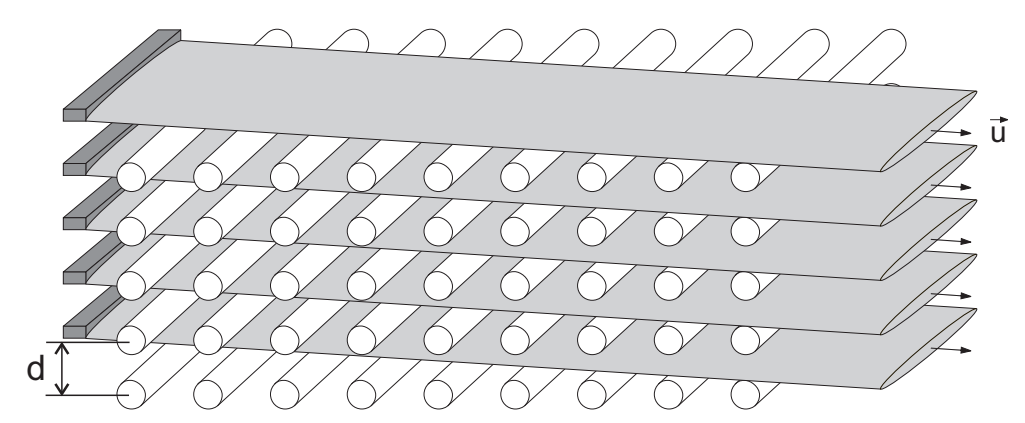

FIG. 1: General view of Volume Free Electron Laser formed by metal threads with several sheet electron beams.

$$
\begin{array}{r}
D E-\omega^{2} \chi_{1} E_{1}-\omega^{2} \chi_{2} E_{2}-\omega^{2} \chi_{3} E_{3}-\ldots=0 \\
-\omega^{2} \chi_{-1} E+D_{1} E_{1}-\omega^{2} \chi_{2-1} E_{2}-\omega^{2} \chi_{3-1} E_{3}-\ldots=0 \\
-\omega^{2} \chi_{-2} E-\omega^{2} \chi_{1-2} E_{1}+D_{2} E_{2}-\omega^{2} \chi_{1-2} E_{1}-\ldots=0,
\end{array}
$$

Set (11)is obtained by the use of Bloch representation for field

$$
\vec{E}(\vec{r} ; \omega)=\sum \vec{E}_{i} \exp \left\{i\left(\vec{k}+\vec{\tau}_{i}\right) \vec{r}\right\}
$$

and permittivity in a spatially-periodic medium $\left(\varepsilon\left(\vec{r}+\vec{a}_{n}, \omega\right)=\varepsilon(\vec{r}, \omega)\right)$

$$
\varepsilon(\vec{r}, \omega)=1+\sum_{\{\tau\}} \chi_{\tau}(\omega) \exp (i \vec{\tau} \vec{r}),
$$

$\vec{\tau}_{i}=\left\{\frac{2 \pi}{a} i_{1} ; \frac{2 \pi}{b} i_{2} ; \frac{2 \pi}{c} i_{3}\right\}$ are the reciprocal lattice vectors, $\vec{a}_{n}=n_{1} \vec{a}+n_{2} \vec{b}+n_{3} \vec{c}$ are the translation vectors of periodic medium, $i_{1} \div i_{3}, n_{1} \div n_{3}$ are the integer numbers. $D_{\alpha}=k_{\alpha}^{2} c^{2}-\omega^{2} \varepsilon+\chi_{\alpha}^{(b)}$ , $\vec{k}_{\alpha}=\vec{k}+\vec{\tau}_{\alpha}$ is the wave vector of diffracted photon, $\chi_{\alpha}^{(b)}$ is the part dielectric susceptibility, caused by the presence of electron beam:

$$
\begin{aligned}
\chi_{\alpha}^{(b)=} & \frac{1}{\gamma}\left(\omega_{b} / \omega\right)^{2}\left(\mathbf{u e}^{\alpha} / c\right)^{2} \times \frac{k_{\alpha}^{2} c^{2}-\omega^{2}}{\left(\omega-\vec{k}_{\alpha} \vec{u}\right)^{2}} \\
& \text { "cold" beam limit, } \\
\chi_{\alpha}^{(b)=} & -\frac{i \sqrt{\pi}}{\gamma}\left(\omega_{b} / \omega\right)^{2}\left(\mathbf{u e}^{\alpha} / c\right)^{2} \times \frac{k_{\alpha}^{2} c^{2}-\omega^{2}}{\delta_{\alpha}^{2}} x_{\alpha}^{t} \exp \left[-\left(x_{\alpha}^{t}\right)^{2}\right] \\
& \text { " hot" beam limit. }
\end{aligned}
$$

$x_{\alpha}^{t}=\left(\omega-\vec{k}_{\alpha} \vec{u}\right) / \sqrt{2} \delta_{\alpha}, \delta_{\alpha}^{2}=\left(k_{\alpha 1}^{2} \Psi_{1}^{2}+k_{\alpha 2}^{2} \Psi_{2}^{2}+k_{\alpha 3}^{2} \Psi_{3}^{2}\right) u^{2}$ and $\vec{\Psi}=\Delta \vec{u} / u$ spread of electron's velocities in a beam. Values of $\chi_{\alpha}^{(b)}$ in (2) are cited for two opposite limits. First case is 
described by the inequality $\left|\omega-\vec{k}_{\alpha} \vec{u}\right| \gg \delta_{\alpha}$ and corresponds to the so called hydrodynamic or "cold" beam limit. In this case all electrons participate in interaction with electromagnetic wave. Kinetic or "hot" beam limit $\left|\omega-\vec{k}_{\alpha} \vec{u}\right| \precsim \delta_{\alpha}$ supposes that only part of electrons participates in interaction process.

As it was mentioned above, synchronism conditions are incompatible with conditions of dynamic diffraction for nonrelativistic and low-relativistic electron beams. In this case generation can appear due to transition radiation in periodic medium and electron beam interacts with slightly coupled component corresponding to the wave vector $\vec{k}_{\tau}=\vec{k}+\vec{\tau}$. According to (11) the dispersion equation, which describes instability of electron beam is as follows:

$$
\left(k^{2} c^{2}-\omega^{2} \varepsilon\right)\left(k_{\tau}^{2} c^{2}-\omega^{2} \varepsilon+\chi_{\tau}^{(b)}\right)-\omega^{2} \chi_{\tau} \chi_{-\tau}=0 .
$$

As synchronism conditions are incompatible with Bragg those, then $k^{2} \neq k_{\tau}^{2}$ in the instability range. At the same time two different types of instability exist depending on radiation frequency. Amplification takes place when electron beam is in synchronism with electromagnetic component $\vec{k}+\vec{\tau}$, which has positive projection $k_{z}$. If projection $k_{z}$ is negative and generation threshold is reached, then generation occurs. In the first case radiation propagates along the transmitted wave, which has positive projection of group velocity $v_{z}=\frac{c^{2} k_{z}^{(0)}}{\omega}\left(k_{z}^{(0)}=\sqrt{\omega^{2} \varepsilon-k_{\perp}^{2}}\right)$, and beam disturbance moves along it. In the second case the group velocity has negative projection $v_{z}=-\frac{c^{2} k_{z}^{(0)}}{\omega}$ and radiation propagates along back-wave and electromagnetic wave comes from the range of the greatest beam disturbance to the place, where electrons come into the interaction area. For one-dimensional structure such mechanism is realized in backward-wave tube.

Dispersion equation, which describes the roots, corresponding interaction of electron beam with electromagnetic wave at $k^{2} \neq k_{\tau}^{2}$ can be rewritten as:

$$
u_{z}^{2}\left(k_{z}-a_{1}\right)^{2}\left(k_{z}-k_{z}^{(0)}\right)\left(k_{z}+k_{z}^{(0)}\right)=-\frac{a \omega_{L}^{2}\left(\vec{u} \vec{e}^{\tau}\right)^{2} \omega^{4} r}{c^{4}\left(k_{\tau}^{2} c^{2}-\omega^{2} \varepsilon_{0}\right)},
$$

where $a_{1}=\frac{\omega-\vec{k}_{\perp} \vec{u}_{\perp}}{u_{z}}-\tau_{z}$. For amplification case (4) gives for increment of instability:

$$
\operatorname{Im} k_{z}^{\prime}=-\frac{\sqrt{3}}{2} f
$$


where $f=\sqrt[3]{\frac{a \omega_{L}^{2}\left(\vec{u}_{e^{T}}\right)^{2} \omega^{4} r}{2 k_{z}^{(0)} c^{4} u_{z}^{2}\left(k_{\tau}^{2} c^{2}-\omega^{2} \varepsilon_{0}\right)}}$, if condition $2 k_{z}^{\prime} f \gg \frac{\omega^{2} \chi 0^{\prime \prime}}{c^{2}}$ is fulfilled. In case $2 k_{z}^{\prime} f \ll \frac{\omega^{2} \chi 0^{\prime \prime}}{c^{2}}$ dissipative instability evolves. Its increment is

$$
I m k_{z}=-\frac{c}{\omega} \sqrt{\frac{k_{z}^{(0)} f^{3}}{\chi_{0}}} .
$$

If inequalities $k_{z}^{\prime 2} \gg 2 k_{z} k_{z}^{\prime}$ and $k_{z}^{\prime 2} \gg \frac{\omega^{2} \chi_{0}^{\prime \prime}}{c^{2}}$ are fulfilled, spatial increment of instability can be expressed as:

$$
\operatorname{Im} k_{z}^{c}=-\left(\frac{a \omega_{L}^{2}\left(\vec{u} e^{T}\right)^{2} \omega^{4} r}{c^{4}\left(k_{\tau}^{2} c^{2}-\omega^{2} \varepsilon_{0}\right) u_{z}^{2}}\right)^{1 / 4}
$$

but parameters providing dependence (7) correspond to the conversion from amplification to generation regime. For Compton instability (at proper electron beam density) this situation takes place at $k_{z}^{(0)} \approx 0$

Frequency of amplified radiation is defined as:

$$
\omega=\frac{\vec{\tau} \vec{u}}{1-\beta_{x} \eta_{x}-\beta_{y} \eta_{y}-\beta_{z} \sqrt{\varepsilon-\eta_{x}^{2}-\eta_{y}^{2}}} .
$$

Instability in generation regime is described by the temporal increment and can not be described by the spatial that. Increment of absolute instability can be found solving the equation

$$
\operatorname{Im}_{z}^{(+)}(\omega)=\operatorname{Im} k_{z}^{(-)}(\omega)
$$

with respect to imaginary part of $\omega$. Roots of (4) are expressed as:

$$
\begin{gathered}
k_{z 1}^{(-)}=a_{1}-\frac{a_{1}+k_{z}^{(0)}}{3}+A+B \\
k_{z 2,3}^{(+)}=a_{1}-\frac{a_{1}+k_{z}^{(0)}}{3}-\frac{A+B}{2} \pm i \frac{A-B}{2} \sqrt{3}
\end{gathered}
$$

where

$$
\begin{aligned}
& A=\sqrt[3]{\frac{f^{3}}{2}-\left(\frac{a_{1}+k_{z}^{(0)}}{3}\right)^{3}+\sqrt{\frac{f^{6}}{4}-f^{3}\left(a_{1}+k_{z}^{(0)}\right)^{3}}} \\
& B=\sqrt[3]{\frac{f^{3}}{2}-\left(\frac{a_{1}+k_{z}^{(0)}}{3}\right)^{3}-\sqrt{\frac{f^{6}}{4}-f^{3}\left(a_{1}+k_{z}^{(0)}\right)^{3}}} .
\end{aligned}
$$




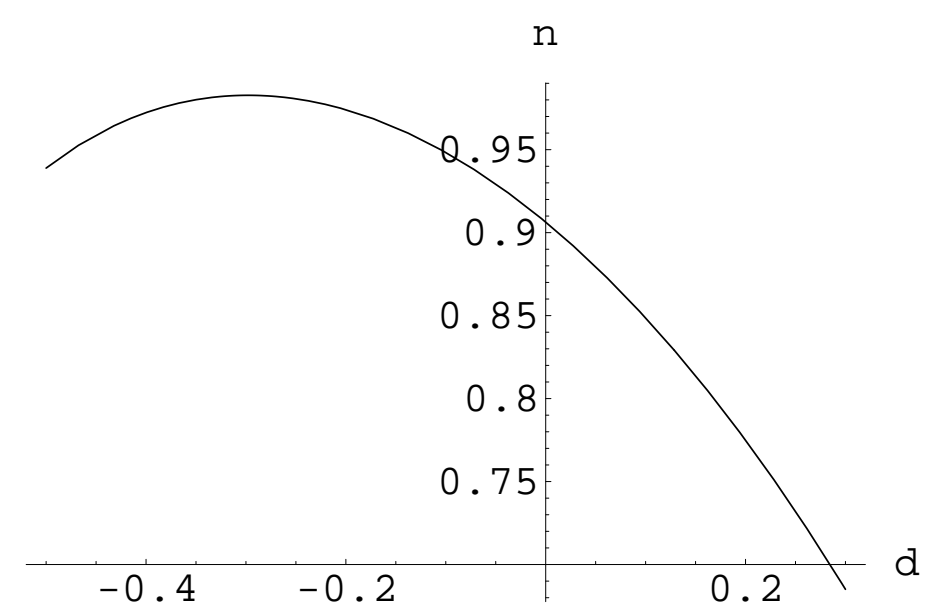

FIG. 2: Calculated dependence of temporal increment on detuning parameter.

Calculated dependence of temporal increment on parameter of detuning is presented in Fig.2.

Axes in Fig.2 2 are denoted as: $d=\frac{a_{1}+k_{z}^{(0)}}{f}, n=\frac{\frac{\omega^{\prime \prime}}{u_{z}}+\frac{2 \omega \omega^{\prime \prime} \varepsilon_{0}+\omega^{2} \chi_{0} \prime}{c^{2} k_{z}^{(0)}}}{f}$. It follows from Fig.2 that at certain value of parameter of detuning:

$$
\frac{a_{1}+k_{z}^{(0)}}{f} \approx-0.3
$$

increment of instability has maximum peak

$$
\frac{\frac{\omega^{\prime \prime}}{u_{z}}+\frac{2 \omega \omega^{\prime \prime} \varepsilon_{0}+\omega^{2} \chi_{0} "}{c^{2} k_{z}^{(0)}}}{f} \approx 0.98 .
$$

Increment of absolute instability can be found from (12). It is easy to see that absolute instability can evolve if current exceed start value, which is determined by dissipation. Amplification regime has no threshold and deciding influence of dissipation causes dissipative instability (6).

Frequencies, corresponding to generation regime, are defined by the expression different from (8):

$$
\omega=\frac{\vec{\tau} \vec{u}}{1-\beta_{x} \eta_{x}-\beta_{y} \eta_{y}+\beta_{z} \sqrt{\varepsilon-\eta_{x}^{2}-\eta_{y}^{2}}} .
$$

Thus, it follows from (8, 13) that change of radiation angle causes smooth frequency tuning. At that, generation frequencies are less than those corresponding to amplification regime. Hence, using system as amplifier one should add dispersion elements in it to raise dissipation in frequency range, in which generation occurs. 
Use of Bragg multiwave distributed feedback increases generation efficiency and provides discrimination of generated modes. If conditions of synchronism and Bragg conditions are not fulfilled simultaneously, diffraction structures with two different periods can be applied [6]. The first of them provides synchronism of electromagnetic wave with electron beam $\omega-\vec{k} \vec{u}=\overrightarrow{\tau_{1}} \vec{u}$, where $\vec{\tau}_{1}$ is the reciprocal lattice vector of this structure. The second diffraction structure evolves distributed Bragg coupling $|\vec{k}| \approx\left|\vec{k}+\vec{\tau}_{j}\right|, \vec{\tau}_{j}(j=2 \div n)$ are the reciprocal lattice vectors of the second structure. Conditions of synchronism and Bragg conditions can be fulfilled even for one diffraction structure, because diffraction structure with one period has unlimited set of reciprocal lattice vectors. Then reciprocal lattice vectors providing synchronism and Bragg conditions are significantly different in value.

It follows from (1) that dispersion equation for distributed feedback supplied by two-wave diffraction with one low coupled wave is expressed as:

$$
F_{3}\left(\vec{k} ; \vec{k}_{1} ; \vec{k}_{2}\right)=-\chi_{1}^{(b)} F_{2}\left(\vec{k} ; \vec{k}_{2}\right)
$$

where:

$$
\begin{aligned}
F_{3}\left(\vec{k} ; \vec{k}_{1} ; \vec{k}_{2}\right)= & \left(k^{2} c^{2}-\omega^{2} \varepsilon\right)\left(k_{1}^{2} c^{2}-\omega^{2} \varepsilon\right)\left(k_{2}^{2} c^{2}-\omega^{2} \varepsilon\right)- \\
& \omega^{4}\left(k^{2} c^{2}-\omega^{2} \varepsilon\right) \chi_{1-2} \chi_{2-1}-\omega^{4}\left(k_{1}^{2} c^{2}-\omega^{2} \varepsilon\right) \chi_{2} \chi_{-2} \\
& -\omega^{4}\left(k_{2}^{2} c^{2}-\omega^{2} \varepsilon\right) \chi_{1} \chi_{-1}-\omega^{6}\left(\chi_{1} \chi_{-2} \chi_{2-1}+\chi_{2} \chi_{-1} \chi_{1-2}\right) \\
F_{2}\left(\vec{k} ; \vec{k}_{2}\right)= & \left(k^{2} c^{2}-\omega^{2} \varepsilon\right)\left(k_{2}^{2} c^{2}-\omega^{2} \varepsilon\right)-\omega^{4} \chi_{2} \chi_{-2} .
\end{aligned}
$$

(14) is derived with the assumption that synchronism conditions are fulfilled for reciprocal lattice vector $\vec{\tau}_{1}\left(\omega-\left(\vec{k}+\vec{\tau}_{1}\right) \vec{u} \approx 0\right)$, while two-wave diffraction evolves at planes with reciprocal lattice vector $\vec{\tau}_{2}\left(|\vec{k}| \approx\left|\vec{k}+\vec{\tau}_{2}\right|\right)$. Threshold conditions of generation and increment of temporal instability for the latter geometry were obtained in [6]:

$$
\omega^{\prime \prime}=\frac{\omega}{2(1-\beta)}\left\{G-\left(\frac{\gamma_{0} u}{\vec{n} \vec{u}}\right)^{3} \frac{16 \pi^{3} n^{2}}{-\beta\left(k \chi_{2} L_{*}\right)^{2} k L_{*}}-\chi_{0}^{\prime \prime}\left(1-\beta \pm \sqrt{-\beta} \frac{r^{\prime \prime}}{\left|\chi_{2}\right| \chi_{0}^{\prime \prime}}\right)\right\}
$$

where: 


$$
\begin{aligned}
G= & \left(k L_{*}\right)^{2} \frac{\pi^{2} n^{2}}{4 \gamma}\left(\frac{\omega_{b}}{\omega}\right)^{2}\left(\frac{\vec{u} \vec{e}_{1}}{\vec{n} \vec{u}}\right)^{2}\left(l_{1}+\chi_{0}\right) \\
& \times \frac{l \chi_{1-2} \chi_{2-1}+l_{2} \chi_{1} \chi_{-1}+\chi_{1} \chi_{-2} \chi_{2-1}+\chi_{2} \chi_{-1} \chi_{1-2}}{l_{1}^{2}} f(y),
\end{aligned}
$$

$l=\frac{k_{0}^{2} c^{2}-\omega^{2} \varepsilon}{\omega^{2}}, l_{1}=\frac{\left(\vec{k}_{0}+\vec{\tau}_{1}\right)^{2} c^{2}-\omega^{2} \varepsilon}{\omega^{2}}, l_{2}=\frac{\left(\vec{k}_{0}+\vec{\tau}_{2}\right)^{2} c^{2}-\omega^{2} \varepsilon}{\omega^{2}}$. Condition $\omega^{\prime \prime}=0$ in (15) defines the start current of generation. Threshold conditions for s-wave diffraction is converted to:

$$
G^{(s)}=\frac{a_{s}^{3}}{\left(k \chi L_{*}\right)^{2 s} k L_{*}}+\chi_{0}^{\prime \prime} b_{s} .
$$

For developed dynamic diffraction, when $k|\chi| L_{*} \gg 1$, either generation start current or length of generation zone at certain current value can be reduced.

Each Bragg condition holds one of free parameters. For example, for certain geometry and electron beam velocity two conditions for 3-wave diffraction entirely determine transverse components of wave vector $k_{x}$ and $k_{y}$, and therefore generation frequency (see (8, 13)). Hence, volume diffraction system provides mode discrimination due to multiwave diffraction.

\section{DISCUSSION.}

The above results affirm that volume diffraction structure provides both amplification and generation regimes even in the absence of dynamic diffraction. In latter case generation evolves with backward-wave similarly backward-wave tube. Frequency in such structure is changed smoothly either at smooth variation of radiation angle (variation of $k_{x}$ and $k_{y}$ ) or at rotation of diffraction grating or electron beam (change of $\vec{\tau} \vec{u}$ )(see (8), (13)). For certain geometry and reciprocal lattice vector amplification corresponds higher frequencies then generation. Rotation of either diffraction grating or electron beam also changes value of boundary frequency, which separates generation and amplification ranges. Use of multiwave distributed feedback owing to Bragg diffraction, let either to increase generation efficiency or to reduce length of interaction area $((15)$, (14)). In this case generation is available with both backward and following waves.

In particular, the proposed volume structure can be used for generation of submillimeter radiation by accelerator LIU-3000. Parameters of this setup: electron beam 
energy $E=800 \mathrm{keV}$, beam current $I=100 \div 200 \mathrm{~A}$. To generate radiation with wavelength $0.3 \mathrm{~mm}$ in such a system volume structure composed from strained threads should has period $\sim 2 \mathrm{~mm}$, and period of diffraction grating providing Bragg coupling is $\sim 0.16 \mathrm{~mm}$.

[1] V.G.Baryshevsky, I.D.Feranchuk Phys.Let 102A,141,(1984).

[2] V.G.Baryshevsky, K.G.Batrakov, I.Ya.Dubovskaya Journ.Phys.D 24,1250,(1991).

[3] V.G.Baryshevsky, K.G.Batrakov, I.Ya.Dubovskaya NIM A 358, 493,(1995).

[4] V.G.Baryshevsky, K.G.Batrakov, I.Ya.Dubovskaya, S.Sytova NIM A 358,508,(1995).

[5] V.G.Baryshevsky, K.G.Batrakov, I.Ya.Dubovskaya, V.A.Karpovich, V.N.Rodionova, NIM A 393, p. 71-75, 1997.

[6] V.G.Baryshevsky, K.G.Batrakov, V.I.Stolyarsky Proceedings of 21 FEL Conference, p. 37-38, 1999. 